\title{
Ornithine decarboxylase is a target for chemoprevention of basal and squamous cell carcinomas in Ptch $1^{+/}$mice
}

\author{
Xiuwei Tang, ${ }^{1}$ Arianna L. Kim, ${ }^{1}$ David J. Feith, ${ }^{2}$ Anthony E. Pegg, ${ }^{2}$ Justin Russo, ${ }^{1}$ Hong Zhang, ${ }^{1}$ \\ Michelle Aszterbaum, ${ }^{3}$ Levy Kopelovich, ${ }^{4}$ Ervin H. Epstein, Jr., ${ }^{3}$ David R. Bickers, ${ }^{1}$ \\ and Mohammad Athar ${ }^{1}$
}

\begin{abstract}
${ }^{1}$ Department of Dermatology, College of Physicians and Surgeons, Columbia University, New York, New York, USA. ${ }^{2}$ Department of Cellular and Molecular Physiology, Penn State College of Medicine, Hershey, Pennsylvania, USA. ${ }^{3}$ Department of Dermatology, University of California San Francisco, San Francisco, California, USA. ${ }^{4}$ Division of Cancer Prevention, National Cancer Institute, Bethesda, Maryland, USA.
\end{abstract}

\begin{abstract}
Solar ultraviolet B (UVB) radiation induces cutaneous ornithine decarboxylase (ODC), the first enzyme in the polyamine-biosynthesis pathway, which drives continued proliferation and clonal expansion of initiated (mutated) cells, leading to tumorigenesis. Therefore ODC is a potentially important target for chemoprevention of basal cell carcinomas (BCCs), the majority of which have mutations in the tumor-suppressor gene known as patched $(P T C H)$. To assess this possibility, we first overexpressed ODC in the skin of Ptch1 ${ }^{+/-}$mice using a keratin 6 (K6) promoter that directs constitutive ODC expression in the outer root sheath of the hair follicle. UVB irradiation of these mice accelerated induction of BCCs as compared with their Ptch1 ${ }^{+/-}$littermates. To further verify the role of ODC in BCC tumorigenesis, we used an antizyme (AZ) approach to inhibit ODC activity in the $\mathrm{Ptch1}^{+/-}$mice. $P t c b 1^{+/-}$mice with $\mathrm{AZ}$ overexpression driven by the $\mathrm{K} 6$ promoter were resistant to the induction of BCCs by UVB. Furthermore, oral administration of the suicidal ODC inhibitor $\alpha$-difluoromethylornithine reduced UVB-induced BCCs in Ptch $1^{+/-}$mice. These results demonstrate the crucial importance of ODC for the induction of BCCs and indicate that chemopreventive strategies directed at inhibiting this enzyme may be useful in reducing BCCs in human populations.
\end{abstract}

\section{Introduction}

Basal cell carcinomas (BCCs) are the most common human cancer, affecting one million Americans each year. It is estimated that one in three Caucasians will develop at least one BCC in his or her lifetime (1-3). Patients affected by the rare dominantly inherited disorder nevoid basal cell carcinoma syndrome (NBCCS), or Gorlin syndrome, develop dozens to hundreds of BCCs as well as various extracutaneous tumors (4). NBCCS patients are known to have PTCH1 mutations (1), and mutations in PTCH1 as well as smoothened (SMO) have been identified in sporadic BCCs $(5-10)$. These genes constitute the major components of the sonic hedgehog (SHH) signaling pathway, which plays a key role in BCC development (11). The receptor for SHH is the product of the tumor-suppressor gene PTCH. The protein PTCH is a negative regulator of SHH signaling and thereby blocks the expression of downstream target genes such as Gli, which drives proliferation and PTCH1 itself (12).

Murine models of skin carcinogenesis induced by ultraviolet B (UVB) have provided important insights into the pathogenesis of squamous cell carcinomas (SCCs) (13). However, understanding of the causation of $\mathrm{BCCs}$ has progressed more slowly because of the lack of a suitable animal model for this tumor. In the past decade, mouse models for BCCs have been developed (12). One of these is

Nonstandard abbreviations used: basal cell carcinoma (BCC); $\alpha$-difluoromethylornithine (DFMO); keratin 5 (K5); nevoid basal cell carcinoma syndrome (NBCCS); ornithine decarboxylase (ODC); antizyme (AZ); outer root sheath (ORS); patched (Ptch); sonic hedgehog (SHH); squamous cell carcinoma (SCC);

ultraviolet B (UVB).

Conflict of interest: The authors have declared that no conflict of interest exists.

Citation for this article: J. Clin. Invest. 113:867-875 (2004). doi:10.1172/JCI200420732. the $P t c h 1^{+/}$- knockout mouse, in which exons 1 and 2 are deleted and a Lac reporter gene is inserted at the deleted site $(14,15)$. The skin of these mice appears grossly normal. However, histologic examination reveals microscopic basaloid cell proliferation that mimics BCCs as well as benign trichoblastomas. Exposure of the skin of these mice to UVB or $\gamma$-ray irradiation accelerates the induction of BCCs (16).

Ornithine decarboxylase (ODC) catalyzes the conversion of ornithine to putrescine, which is the essential building block required for the production of higher polyamines (spermidine and spermine) within the cell. Polyamines are ubiquitous polycationic molecules that are critical for normal and neoplastic cell growth (17). The importance of ODC in skin tumor development has been investigated in several mouse models (18). Increased levels of ODC play a crucial role in promoting SCCs by driving the continued proliferation and selective clonal expansion of $\mathrm{v}$-Ha-ras-initiated cells (19). Using a similar treatment protocol, it has been shown that ODC activity is higher in BCCs than in normal human skin (20). Virtually all known tumor promoters are potent inducers of ODC (21). Engineered ODC overexpression enhances the tumorigenicity of premalignant epidermal cells that possess an activated Ha-ras gene (22). ODC transgenic mice with keratin 6 promoter-driven overexpression of epidermal ODC (K6-ODC mice) develop squamous papillomas following topical application of sub-threshold doses of carcinogens in the absence of further application of tumor promoter (23). When a similar treatment protocol was used in K6-ODC mice on an FVB genetic background, the majority of tumors that developed were SCCs rather than papillomas (24). UVB rapidly induces papillomas and SCCs in transgenic mice with keratin 5 promoter-driven overexpression of ODC compared with non- 
transgenic littermates (25). It is unclear whether these mechanisms of tumor promotion that are pertinent to induction of SCCs are relevant to BCCs, although, as mentioned earlier, ODC activity is increased in BCCs $(20,26,27)$. Cellular polyamine content is tightly regulated by various mechanisms, including transcriptional, translational, and posttranslational regulation of the enzymes responsible for polyamine synthesis and catabolism as well as a highly inducible polyamine-transport system $(18,28)$. A multifunctional protein known as antizyme (AZ) exerts posttranslational control of ODC $(29,30)$. Overexpression of $A Z$ in the skin of transgenic mice with either keratin 5 (K5) or keratin $6(\mathrm{~K} 6)$ promoter elements leads to decreased ODC activity and polyamine content following cutaneous application of tumor promoters (31).

In this study we have assessed ODC as a molecular target for the chemoprevention of BCCs. ODC overexpression in $P t c h 1^{+/-}$mice augmented UVB induction of BCCs, whereas ODC inhibition both genetically and pharmacologically diminished UVB-induced BCCs, indicating that chemoprevention strategies directed against ODC and cellular polyamines could be useful in reducing the risk of BCCs in human populations.

\section{Methods}

Reagents. Rabbit AZ antiserum was produced at Cocalico Biologicals Inc. (Reamstown, Pennsylvania, USA) using a purified polyhistidinetagged AZ fusion protein (31) and was further purified with a 6xHis-AZ affinity column (AminoLink Plus Immobilization Kit; Pierce Biotechnology Inc., Rockford, Illinois, USA). In addition, commercially obtained antibodies were employed for the Western blot analysis and/or immunohistochemistry using vendors' protocols. Antibodies for cyclins A/D1, Gli1, Ptch1, and Hip were purchased from Santa Cruz Biotech Inc. (Santa Cruz, California, USA), anti-cyclin B1 and anti-K17 antibodies from NeoMarkers (Fremont, California, USA), anti-PCNA antibody from Oncogene (Boston, Massachusetts, USA), and anti-ODC antibody from Sigma-Aldrich (St. Louis, Missouri, USA). Anti-AZ antibody was a gift from Anthony Pegg.

Animals. Ptch $1^{+/-}$heterozygous knockout C57BL/6 mice were developed by deletion of exons 1 and 2 and insertion of the LacZ gene at the deletion site as described previously $(15,16)$. We purchased male breeders (6-7 weeks old) of hemizygous ODC transgenic B6.Cg-Tg (K6-Odc)55Tgo strain (Taconic, Germantown, New York, USA). Transgenic mice with the bovine cytokeratin 6 promoter directing expression of $A Z$ in the skin were derived as described previously (31). The studies presented here used mice with the K6-AZ line 52 transgene on $\mathrm{B} 6 \mathrm{C} 3 \mathrm{~F} 2$ backcrossed onto the $\mathrm{C} 57 \mathrm{BL} / 6 \mathrm{~J}$ inbred strain for seven generations or more (31). The detailed breeding protocols and genotyping of $\mathrm{Ptch}^{+/-}$heterozygous ODC transgenic and $P t c h 1^{+/-}$heterozygous ODC AZ transgenic animals were described previously $(15,31)$. The animal experiments were conducted using protocols approved by the institutional review board.

UV light source. A UV irradiation unit (Daavlin Co., Bryan, Ohio, USA) equipped with an electronic controller to regulate dosage was used routinely for these studies. The UVB source consisted of eight FS72T12-UVB-HO lamps that emitted UVB (290-320 nm, 75-80\% of total energy) and ultraviolet A (320-380 nm, 20-25\% of total energy). We used a cellulose film (Kodacel TA401/407) (Eastman Kodak Co., Rochester, New York, USA) to eliminate ultraviolet C (UVC) radiation. A UVC sensor (Goldilux UVC probe; Thermo Oriel, Stratford, Connecticut, USA) was used during each exposure to confirm lack of UVC emission. The dose of UVB was quantified with a UVB Spectra 305 dosimeter (Daavlin Co.). The radiation was further cal- ibrated with an IL1700 research radiometer/photometer from International Light Inc. (Newburyport, Massachusetts, USA). The distance between the radiation source and targets was maintained at 30 $\mathrm{cm}$. The irradiation assembly was kept in an air-conditioned room and a fan was placed inside the exposure chamber to minimize temperature fluctuations during irradiation as described previously (32).

Assessment of microscopic lesions. Microscopic BCC-like lesions were defined as tumor islands composed of monomorphic basaloid cells with scant cytoplasm arranged as nests within the dermis. These lesions were counted as numbers per unit area and also as total tumor area per square millimeter skin section. Skin samples were prepared using $10 \%$ buffered formalin-fixed $H \& E$-stained and $\beta$-gal-stained samples of full-thickness dorsal skin. For $\beta$-gal staining, glutaraldehyde and formalin-fixed tissue were treated with $\mathrm{X}$-gal and iron buffer solution (Roche Diagnostics Corp., Indianapolis, Indiana, USA) for 48 hours and processed using the vendor's protocol.

$R N A$ isolation and RT-PCR. Total cellular RNA was isolated from control skin and BCCs as described previously (16). RT-PCR was carried out using a Promega A-1260 kit (Promega Corp., Madison, Wiscon$\sin$, USA) according to the vendor's protocol. A list of primers used to assess the expression of SHH pathway genes in skin and UVBinduced tumors in $\mathrm{Ptch}^{+/-} / \mathrm{ODC}$ TgN mice is shown in Table 1.

The methods of scoring of carcinoma incidence and multiplicity, H\&E staining, immunohistochemical analysis of Gli1, Ptch, hip, cyclin D1, and PCNA proteins, tissue lysate preparation, Western blotting, and measurement of ODC activity and polyamine levels were the same as published previously $(16,20,21,27,31,32)$.

Experimental protocol. To investigate the effects of ODC overexpression on the induction of BCCs by UVB in $P t c h 1^{+/-}$mice, seven of the 14 first-generation $P t c h 1^{+/} /$ODC TgN mice were UVB-irradiated (180 $\mathrm{mJ} / \mathrm{cm}^{2}$ twice per week for 30 weeks beginning at week 8 ), and the remaining seven were sham-irradiated and served as control. At the end of 30 weeks, all animals were killed; their dorsal skin was excised, cut into small pieces with surgical scissors, and used for $\beta$-gal staining, histology, immunohistochemistry, and epidermal preparations for RT-PCR. The dose of $180 \mathrm{~mJ} / \mathrm{cm}^{2}$ was selected because, in our experience, it is consistently tumorigenic in SKH-1 hairless mice (33).

To assess the effects of tissue depletion of ODC and polyamines on UVB-induced BCCs, we used $P t c h 1^{+/-}$mice overexpressing AZ as well as their littermates, which included $P t c h 1^{+/}, P t c h 1^{+/+}$, and $P t c h 1^{+/+} / \mathrm{AZ}$ mice generated by crossing of the K6-AZ mice with our

\section{Table 1}

Primers used to assess the expression of SHH pathway genes

$\begin{array}{ll}\text { Genes } & \text { Primers } \\ \text { Ptch1 } & \text { 5'-AACAAAAATTCAACCAAACCTC-3' } \\ & \text { 5'-TGTCTTCATTCCAGTTGATGTG-3' } \\ \text { Ptch2 } & \text { 5'-TGCCTCTCTGGAGGGCTTCC-3' } \\ & \text { 5'-CAGTTCCTCCTGCCAGTGCA-3' } \\ \text { Gli1 } & \text { 5'-GTCGGAAGTCCTATTCACGC-3' } \\ & \text { 5'-CAGTCTGCTCTCTTCCCTGC-3' } \\ \text { Gli2 } & \text { 5'-GAGCAGAAGCCCTTCAAG-3' } \\ & \text { 5'-GACAGTCTTCACATGCTT-3' } \\ \text { Gli3 } & \text { 5'-CAAGCCTGATGAAGACCTCC-3' } \\ & \text { 5'-GCTTTGAACGGTTTCTGCTC-3' } \\ \text { Cyclin D1 } & \text { 5'-CTCTGGCTCTGTGCCTTTCT-3' } \\ & \text { 5'-CCGGAGGACTCAGAGCAAATC-3' } \\ \text { Actin } & \text { 5'-TACCACAGGCATTGTGATGGA-3' } \\ & \text { 5'-CAACGTCACACTTCATGATGG-3' }\end{array}$



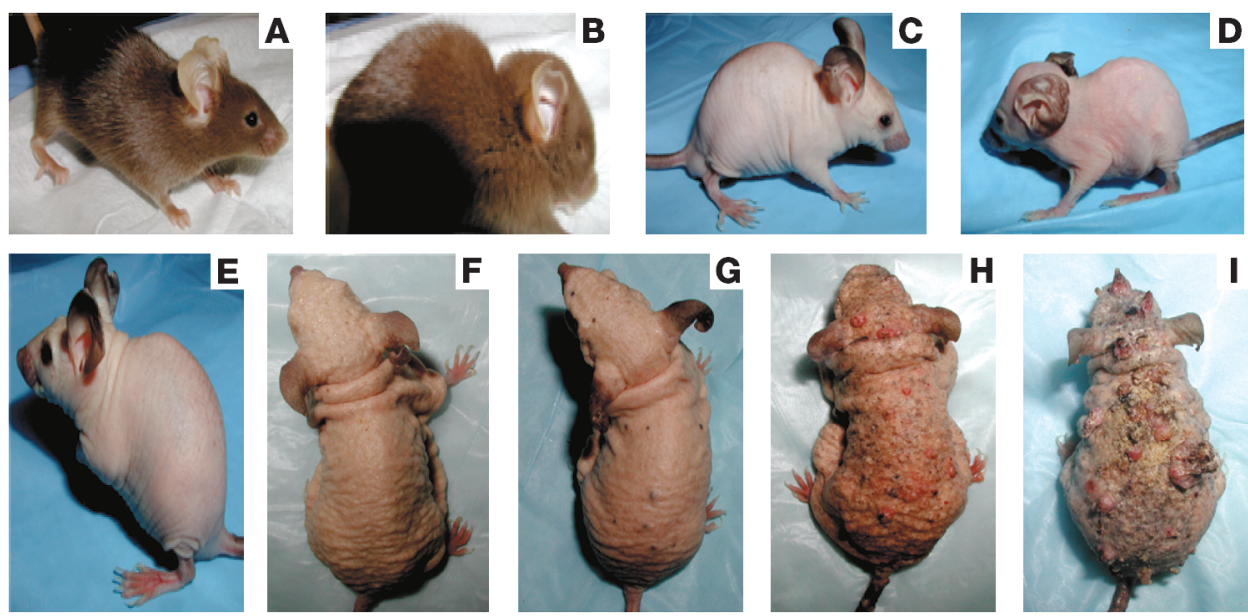

H

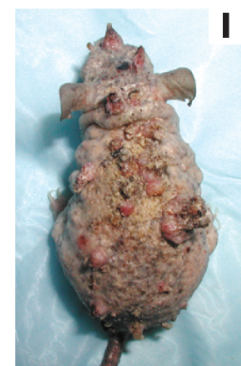

\section{Figure 1}

Ptch1+/- heterozygous mice overexpressing ODC (Ptch1+/-/ODC TgN mice). (A) Ptch $1^{+/-}$heterozygous mouse. (B) Ptch $1^{+/-}$ heterozygous mouse showing skull abnormality. (C) Ptch1+/-/ODC TgN mouse. (D) Ptch1+-/ODC TgN mouse showing skull abnormality. (E) Ptch1+/-/ODC TgN mouse at week 10. (F) Ptch1+/-/ODC TgN mouse at week 30. (G) UVB-irradiated Ptch 1+l-1 ODC TgN mouse at week 10. (H) UVBirradiated Ptch1+/-/ODC TgN mouse at week 20. (I) UVB-irradiated Ptch1+//ODC $\mathrm{TgN}$ mouse at week 30 .
$\mathrm{Ptc1}^{+/-}$mice. These four groups of mice (ten mice in each group) were UVB-irradiated $\left(240 \mathrm{~mJ} / \mathrm{cm}^{2}\right.$ three times per week for 37 weeks), and tumor data were recorded weekly. The dose of $240 \mathrm{~mJ} / \mathrm{cm}^{2} \mathrm{UVB}$ is based on previously published studies showing that this dose is sufficient to induce both microscopic and visible tumors in $\mathrm{Ptch} 1^{+/-}$ mice (16). After 37 weeks, all animals were killed; their dorsal skin was excised, cut into small pieces, and used for $\beta$-gal staining, histologic examination, immunohistochemistry, and preparation of whole skin lysates for assays of polyamine levels and ODC activity.

To investigate whether pharmacologic inhibition of ODC can reduce the growth of UVB-induced BCCs, we used $\alpha$-difluoromethylornithine (DFMO), a known potent inhibitor of ODC (34), and administered it to UVB-irradiated $P t c h 1^{+/}$mice. For this experiment, $60 \mathrm{Ptch}^{+/-}$mice were irradiated with UVB $\left(240 \mathrm{~mJ} / \mathrm{cm}^{2}\right.$ three times per week) for 32 weeks. Thereafter, UVB irradiation was discontinued, and tumor-bearing animals were divided into two groups of 20 each in such a way that initially each group had approximately the same number of tumors. Non-tumor-bearing UVB-irradiated mice were not used. Group 1 animals were then given ordinary drinking water for the next 20 weeks, whereas group 2 mice received 1\% DFMO in drinking water. The tumor data were recorded weekly and plotted as number of tumors per mouse as a function of weeks on treatment. The mice were sacrificed at 52 weeks, their dorsal skin was removed, and tumors were harvested and collected for Western blot, RT-PCR, $\beta$-gal staining, and histologic and immunohistochemical studies.

\section{Results}

\section{Studies in Ptch1 ${ }^{+/}$beterozygous mice overexpressing ODC}

$\mathrm{Ptch}^{+/-}$heterozygous mice overexpressing ODC. We generated $\mathrm{Ptch}^{+/-}$mice expressing the K6-ODC transgene, hereafter referred to as Ptch $1^{+/-}$ODC TgN. Fourteen Ptch1 $1^{+/} /$ODC TgN mice (including both

\section{Figure 2}

Effects of ODC overexpression on the development of UVB-induced tumors in the skin of Ptch1+- mice. (A) Effects of ODC overexpression on the number of UVB-induced tumors. $P<0.002$ vs. Ptch $1^{+-}$mice. (B) Effects of ODC overexpression on the area of UVB-induced microscopic BCC-like lesions. $P<0.043$ vs. Ptch $1^{+/}$mice. (C) Effects of ODC overexpression on the number of UVB-induced microscopic BCC-like lesions. $P<0.024$ vs. Ptch $1^{+/-}$mice. (D) Histologic staining for $\beta$-gal (blue) in trichoblastoma-like lesions (i) and BCC-like lesions (ii).

males and females) were used. ODC overexpression in $P t c h 1^{+/-}$mice yielded a hairless phenotype similar to that of the parent ODC transgenic animals $(23,35)$. These mice were fertile but smaller in birth weight than their $P t c h 1^{+/-}$nontransgenic littermates and showed excessive nail growth and a normal first hair cycle, followed by progressive hair loss beginning at 2-3 weeks of age. With increasing age, the skin manifested pronounced wrinkling and folding. Similar to Ptch $1^{+/-}$mice (Figure 1B), some of these animals exhibited developmental abnormalities (Figure 1D), including skull deformities. The skin of untreated animals was grossly normal. However, histologic examination of the skin showed small basaloid lesions (data not shown). Immunohistochemical studies of these sections verified that these BCC-like lesions stain positively for Gli1, $\beta$-gal, and hip (data not shown). None of the $P t c h 1^{+/+}$K6-ODC or $P t c h 1^{+/-}$nontransgenic littermates demonstrated such lesions or showed positive staining for $\beta$-gal.

UVB-induced BCCs. We evaluated the effect of chronic UVB irradiation $\left(180 \mathrm{~mJ} / \mathrm{cm}^{2}\right.$ twice per week for 30 weeks beginning at week 8) on the induction of BCCs in Ptch1 $1^{+/} /$ODC TgN mice. Following 10 weeks of UVB exposure, multiple small visible tumors were apparent, and they increased in both number and size during the following 20 weeks of irradiation, reaching a tumor incidence of $100 \%$ by week 30 . At this

A

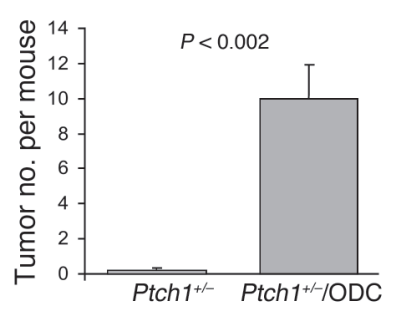

C

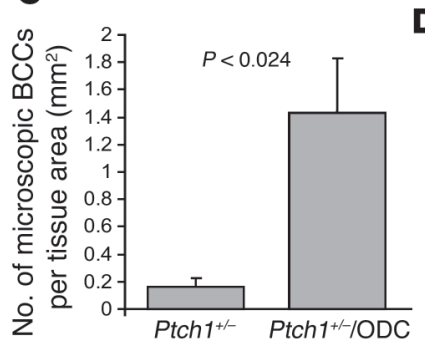

B

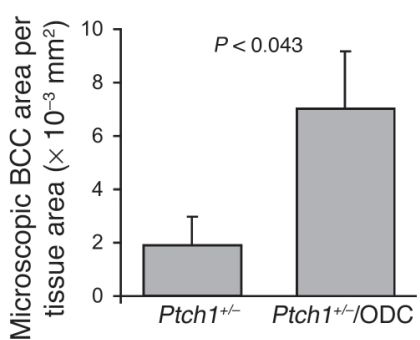

D

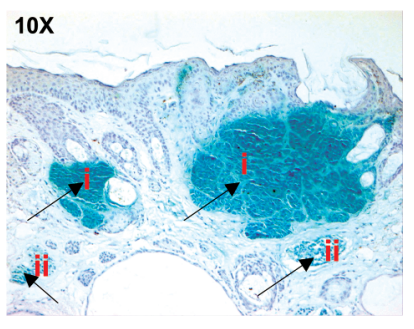


Table 2

Effects of AZ expression on UVB-induced tumorigenesis in Ptch1+-- and wild-type $($ Ptch1+/+) mice

\begin{tabular}{lccccc} 
Groups & $\begin{array}{c}\text { Total no. of } \\
\text { mice analyzed }\end{array}$ & BCCs & \multicolumn{4}{c}{ Tumors/mouse } \\
SCCs & Papillomas & Fibrosarcomas \\
Ptch $h^{+/}$ & 14 & $0.2 \pm 0.11 \mathrm{~A}$ & $0.2 \pm 0.11^{\mathrm{B}}$ & 0 & $0.60 \pm 0.13^{\mathrm{C}}$ \\
Ptch $^{+/ /} / \mathrm{AZ}$ & 12 & 0 & 0 & $0.17 \pm 0.1$ & 0 \\
Ptch $^{+/+}$ & 11 & 0 & $0.36 \pm 0.15^{\mathrm{D}}$ & 0 & $0.36 \pm 0.15^{\mathrm{E}}$ \\
Ptch $^{+/ /} / \mathrm{AZ}$ & 12 & 0 & 0 & $0.17 \pm 0.1$ & 0
\end{tabular}

Each data point represents mean \pm SEM. ${ }^{A} P<0.04,{ }^{B} P<0.04,{ }^{C} P<0.001$ vs. $P t c h^{+1 /} / A Z$. $\mathrm{DP}<0.02, \mathrm{E} P<0.08$ vs. $P$ tch ${ }^{+/+} / \mathrm{AZ}$.

time, innumerable tumors were visible on the dorsal surface of virtually all of these mice (Figure 1I). As shown in Figure 2A, the number of tumors per mouse was $10 \pm 1.9$ (only tumors greater than $4 \mathrm{~mm}$ in diameter were counted). Histologically, many of these lesions resembled human BCCs and/or trichoblastomas, with tumor islands consisting of monomorphic basaloid cells with scant cytoplasm arranged as nests within the dermis. Immunohistochemical studies of skin sections of these mice showed interfollicular basaloid proliferation and histologic features of BCCs and trichoblastomas. The area (Figure 2B) and number (Figure $2 \mathrm{C}$ ) of microscopic BCCs that developed following 30 weeks of UVB exposure were $7.05 \times 10^{-3} \pm 2.1 \times 10^{-3} \mathrm{~mm}^{2}$ and $1.43 \pm 0.4 / \mathrm{mm}^{2}$ tissue area, respectively, which were significantly higher $(P<0.04)$ than the tumor burden in their $P t c h 1^{+/-}$littermates. Both BCCs and trichoblastomas showed strong positive staining for $\beta$-gal (Figure 2D), Gli1, and hip. Lesions with strong $\beta$-gal staining also stained positive for Gli1 (data not shown). It is of interest that some lesions in UVB-irradiated skin of $P t c h 1^{+/} /$ODC TgN mice resembled UVB-induced murine SCCs. RT-PCR analysis of total RNA from dorsal skin showed high expression of Gli1, Gli2, Ptch1, and Ptch2 in UVB- induced BCCs and adjacent nontumor skin compared with age-matched nonirradiated control skin. However, Gli3 expression was similar in tumor and control skin. We next assessed the tissue distribution of ODC expression in control (nonirradiated) and UVB-irradiated nontumor skin and BCC-bearing skin of these mice. ODC expression was localized predominantly in the suprabasal epidermis in UVB-irradiated nontumor skin (data not shown). Interestingly, intense staining for ODC occurred in the stroma surrounding BCCs. Staining in the tumor stroma may be related to UVB-induced ODC. In contrast, spontaneously developing BCC-like lesions revealed no stromal staining (data not shown).

Molecular characterization of UVB-induced BCCs. Cyclin D1 overexpression has been documented in human BCCs (20). To determine whether murine BCCs also show high expression of cyclin D1, we immunostained paraffin sections of control skin (nonirradiated), nontumor UVB-irradiated skin, and BCCs harvested from Ptch1+/-/ODC TgN mice. Cyclin D1 overexpression was seen in the cytoplasm of suprabasal keratinocytes in UVB-exposed nontumor skin. Furthermore, nuclear staining occurred in most BCCs (data not shown). We also assessed the expression of PCNA, an auxiliary protein of DNA polymerase, as an approach to identifying proliferating cells in control (nonirradiated), nontumor UVB-irradiated skin and BCCs in Ptch1 $1^{+/} / \mathrm{ODC}$ TgN mice. PCNA-positive nuclei were seen in the suprabasal layers of UVBexposed nontumor skin (data not shown). Interestingly, BCCs showed strong immunostaining for PCNA in the tumor margins. In an effort to further document the proliferative properties of these BCCs, we immunostained tumor tissue with an antibody specific for keratin 17, an outer root sheath (ORS) protein. As in human BCCs and BCCs in Gli2 transgenic mice, keratin 17 expression was increased in these BCCs (36). We also immunostained BCCs and uninvolved skin for keratins

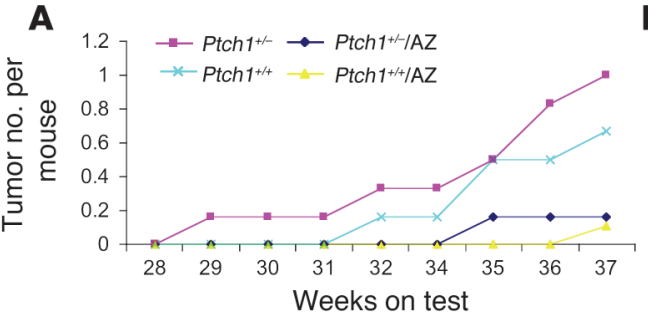

D

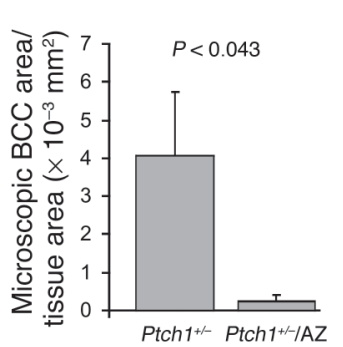

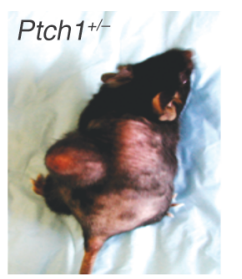

E

Figure 3

Effects of AZ overexpression on the development of UVB-induced tumors in the skin of wild-type and Ptch1+/- mice. (A) Effects of AZ overexpression on the number of UVB-induced tumors. (B) Wild-type and Ptch1+/- normal and AZ-overexpressing mice chronically exposed to UVB. (C) Volume of UVB-induced macroscopic tumors. ${ }^{*} P<0.008$ vs. Ptch $1{ }^{+/-}$. ${ }^{\star \star} P<0.05$ vs. Ptch $1^{+/+}$. (D) Effects of AZ overexpression on the area of UVBinduced microscopic BCC-like lesions. (E) Effects of AZ overexpression on the number of UVB-induced microscopic BCC-like lesions. (F) Histology of skin showing staining for $\beta$-gal (blue) in BCC-like lesions. 
A

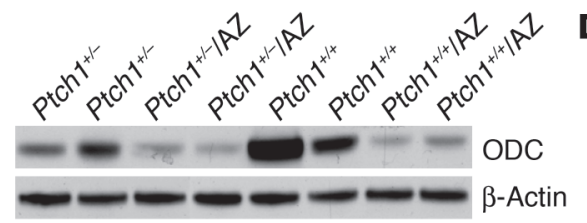

B

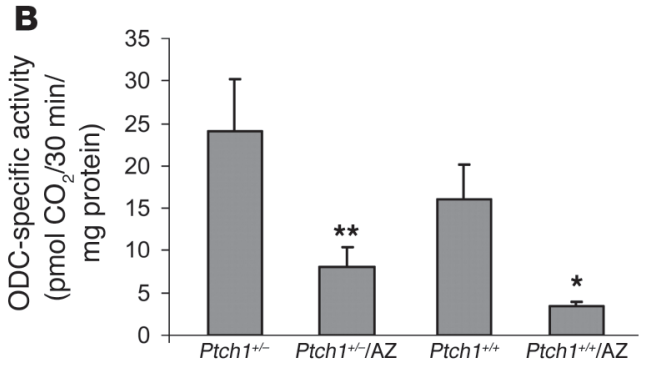

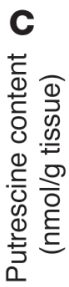

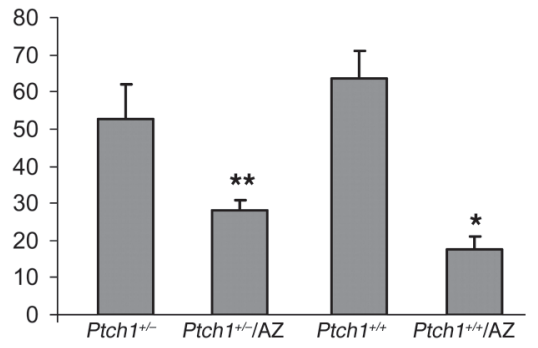

D
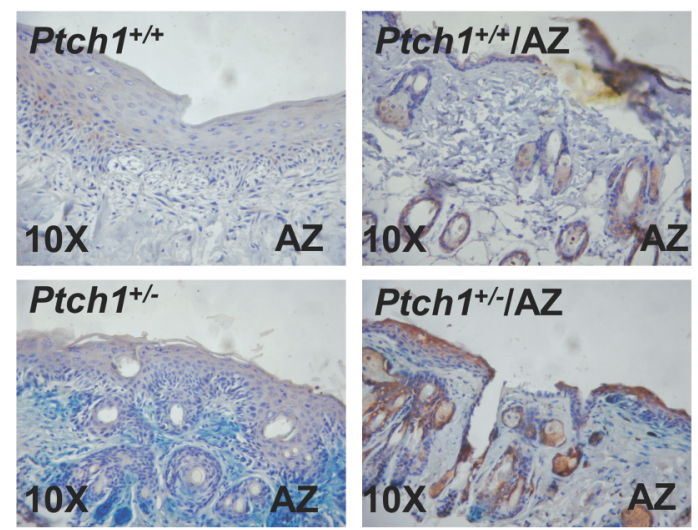

Ptch1+/IAZ

$\mathbf{E}$
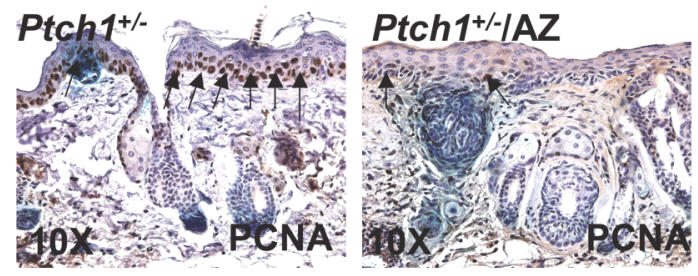

Figure 4

Effects of AZ overexpression on the levels and activity of ODC in the skin of UVB-irradiated wild-type and Ptch1 ${ }^{+/-}$mice. (A) Western blot analysis showing effects of AZ overexpression on ODC. (B) Effects of AZ overexpression on ODC activity. ${ }^{*} P<0.013$ vs. Ptch $1^{+/+}$. ${ }^{* \star} P<0.048$ vs. Ptch $1^{+/-}$. (C) Effects of $A Z$ overexpression on putrescine. ${ }^{*} P<0.005$ vs. Ptch $1^{+/+}$. ${ }^{* *} P<0.046$ vs. Ptch $1^{+/-}$. (D) Immunohistochemical staining for AZ in epidermis of UVB-irradiated Ptch1+/- mice overexpressing AZ. The brown staining in epidermis and hair follicles represents AZ expression. (E) Immunohistochemical staining for PCNA in epidermis and in BCC-like lesions in UVB-irradiated Ptch1+-- mice overexpressing AZ. The dark brown staining in the basal cell layer of epidermis and in tumor islands represents PCNA expression.

1 and 10, known to be markers of terminal differentiation. These keratins were abundantly expressed in suprabasal epidermis of uninvolved skin, but very little or no staining was seen in these BCCs (data not shown). Consistent with the pattern seen in human BCCs, K6 expression was seen in all epidermal layers and in BCCs. K6 expression is characteristic of hyperproliferation and is a feature of tumor cells in BCCs and epidermis overlaying tumor tissue (data not shown).

\section{Studies in Ptch1 ${ }^{+/-}$heterozygous mice overexpressing AZ}

To further demonstrate the importance of ODC induction in BCC development, we used transgenic mice with targeted $\mathrm{AZ}$ expression to inhibit ODC activity and suppress polyamine levels in skin of our $\mathrm{Ptch}^{+/-}$mice and then performed studies to assess their susceptibility to UVB-induced photocarcinogenesis.

Ptch $1^{+/-}$heterozygous mice overexpressing $A Z$. In these experiments, we generated similar numbers of $P t c h 1^{+/-} / \mathrm{AZ} \mathrm{TgN}, P t c h 1^{+/-}, P t c h 1^{+/+}$, and $P t c h 1^{+/+} / A Z$ mice. $P t c h 1^{+/-}$heterozygous mice overexpressing AZ (Ptch1 $1^{+/-} / \mathrm{AZ} \mathrm{TgN}$ mice) have a grossly normal phenotype.

UVB-induced BCCs in Ptch $1^{+/-} / A Z$ TgN mice. The effect of chronic UVB irradiation on $P t c h 1^{+/-} / \mathrm{AZ}$ TgN $, P t c h 1^{+/-}, P t c h 1^{+/+} / \mathrm{AZ} \mathrm{TgN}$, and $P t c h 1^{+/+}$ (wild-type) mice is shown in Figure 3A. These animals developed many visible tumors (Figure 3B). Histologically, these tumors were heterogeneous and included BCCs, SCCs, papillomas, and fibrosarcomas, as shown in Table 2. Ptch1 $1^{+/} / \mathrm{AZ}$ TgN mice developed significantly fewer visible BCCs $(P<0.04)$ compared with their $P t c h 1^{+/}$littermates (Table 2), and the volume of these tumors was also substantially reduced $(P<0.008)$, as shown in Figure 3C. Similarly, tumor area $\left(0.234 \times 10^{-3} \pm 0.16 \times 10^{-3} \mathrm{~mm}^{2}\right.$ vs. $4.059 \times 10^{-3} \pm 1.7 \times 10^{-3} \mathrm{~mm}^{2}$ per unit section area) and number $\left(0.18 \pm 0.04 / \mathrm{mm}^{2}\right.$ tissue area vs. $0.06 \pm$ $0.02 / \mathrm{mm}^{2}$ tissue area) of microscopic BCCs were also significantly reduced $(P<0.04)$ in the $P t c h 1^{+/-} / \mathrm{AZ}$ TgN mice as compared with the $P t c h 1^{+/-}$mice, as shown in Figure 3, D and E, respectively. The histology and relative size of microscopic BCCs in $P t c h 1^{+/}$and $P t c h 1^{+/-} / \mathrm{AZ} \mathrm{TgN}$ mice are shown in Figure 3F. Ptch $1^{+/+} / \mathrm{AZ} \mathrm{TgN} \mathrm{mice} \mathrm{developed} \mathrm{no} \mathrm{SCCs}$ or fibrosarcomas and only a few papillomas, whereas their $P t c h 1^{+/+}$littermates developed mainly SCCs and fibrosarcomas (Table 2). Ptch $1^{+/-} / \mathrm{AZ}$ $\mathrm{TgN}$ mice also manifested only papillomas. However, like $P t c h 1^{+/+}$mice, Ptch $1^{+/-}$mice developed SCCs and fibrosarcomas (Table 2).

Effects of UVB irradiation on cutaneous ODC and polyamines. The effects of UVB on the induction of ODC in skin of $P t c h 1^{+/-} / \mathrm{AZ} \mathrm{TgN,}$ $P t c h 1^{+/-}, P t c h 1^{+/+}$, and $P t c h 1^{+/+} / \mathrm{AZ}$ TgN mice are compared in Figure 4. Western blot analysis showed lower ODC levels in $P t c h 1^{+/-} / \mathrm{AZ}$ TgN mice than in $P t c h 1^{+/-}$mice. Similarly, ODC was diminished in $P t c h 1^{+/+} / \mathrm{AZ}$ mice as compared with $P t c h 1^{+/+}$animals (Figure 4A). Parallel to this, UVB induction of ODC activity was substantially lower in $P t c h 1^{+/-} / \mathrm{AZ}$ TgN mice than in $P t c h 1^{+/-}$mice $(P<0.05)$. Similarly, ODC induction was much lower in $P t c h 1^{+/+} / \mathrm{AZ}$ mice than in their Ptch $1^{+/+}$littermates $(P<0.013)$, as shown in Figure 4B. Next we assessed the effects of AZ overexpression on UVB-induced polyamine synthesis during BCC tumorigenesis. Overexpression of AZ in both UVB-irradiated $P t c h 1^{+/-}$and $P t c h 1^{+/+}$mice significantly diminished putrescine levels (Figure 4C). Spermidine and spermine 
A

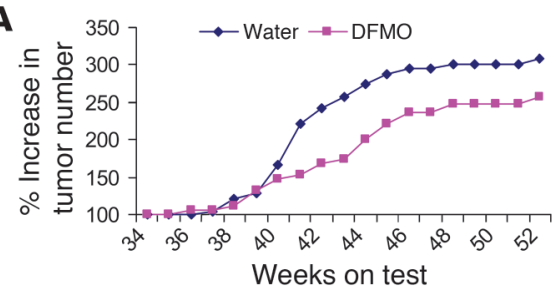

B
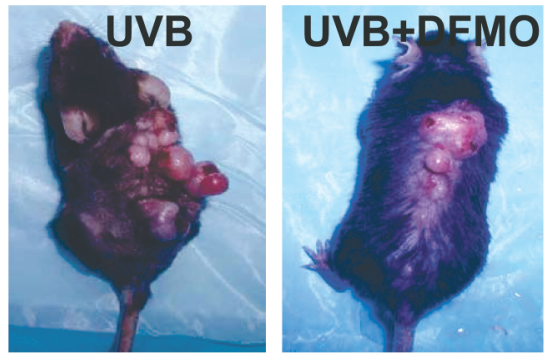

C

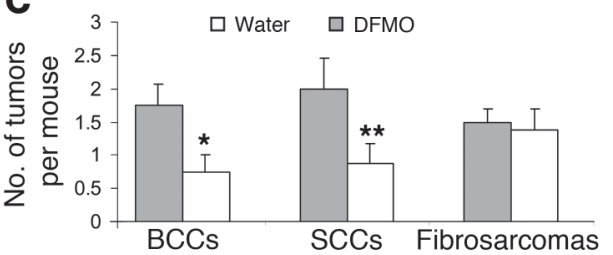

D
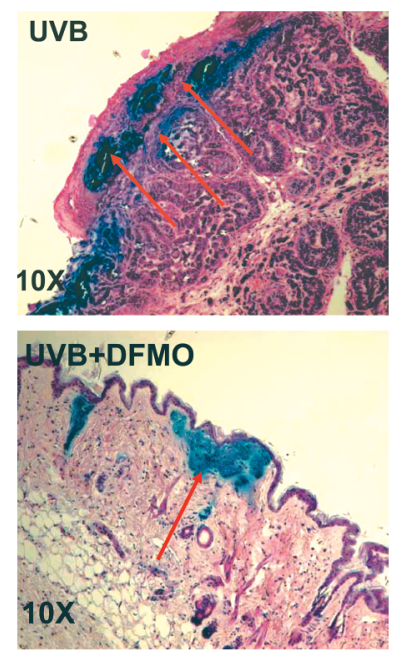

E

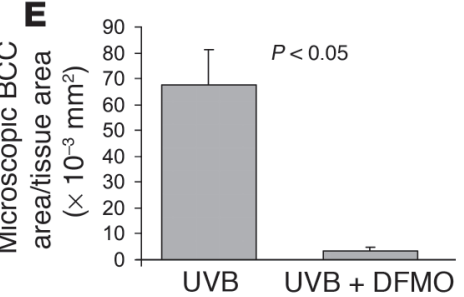

\section{Figure 5}

Effect of DFMO on UVB-induced skin tumorigenesis in Ptch 1+/- mice. (A) Effect of DFMO administration on the growth of UVB-induced skin tumors. The experiment was terminated at week 52. (B) Mice showing the effect of DFMO treatment on UVBinduced skin tumors. Left: UVB-treated control. Right: mouse treated with UVB plus DFMO, shown at week 52. (C) Effect of orally administered DFMO on the growth of UVB-induced BCCs, SCCs, and fibrosarcomas. ${ }^{*} P<0.025$. ${ }^{\star \star} P<0.05$. (D) $\beta$-Gal (blue) and $\mathrm{H} \& \mathrm{E}$ (red) staining showing the effect of orally administered DFMO on UVB-induced microscopic BCClike lesions in Ptch1+/- mice. Upper panel: skin section from UVB-irradiated Ptch1+/- mouse. Lower panel: skin section from UVB-irradiated DFMO-treated Ptch 1+/- mouse. (E) Effect of orally administered DFMO on UVB-induced microscopic BCC-like lesions in Ptch1+/- mice. Samples of full-thickness dorsal skin were fixed in $10 \%$ buffered formalin and stained with $\mathrm{H} \& \mathrm{E}$ and $\beta$-gal, and microscopic BCClike lesions were measured as total tumor area per square millimeter skin sample. are additional polyamines formed from putrescine by the sequential action of spermidine synthase and spermine synthase, which catalyzes the transfer of an aminopropyl group from decarboxylated $S$-adenosylmethionine produced by the enzyme $S$-adenosylmethionine decarboxylase $(28,37)$. The level of these polyamines was unaffected (data not shown).

Effects of UVB irradiation on $A Z$ expression. In Ptch1 $1^{+-} / \mathrm{AZ} \mathrm{TgN}$ mice, the K6 promoter directs constitutive AZ expression in the ORS of the hair follicle. In addition, tumor promoters and other proliferative stimuli are known to elevate K6-driven expression of genes in the ORS and to induce their expression in the interfollicular epidermis (38). Immunohistochemical localization studies using specific antibodies directed against $\mathrm{AZ}$ reveal positive staining in hair follicles and some staining in epidermis of UVB-treated $P t c h 1^{+/-} / \mathrm{AZ}$ $\operatorname{TgN}$ and $P t c h 1^{+++} / \mathrm{AZ} \mathrm{TgN} \mathrm{mice} \mathrm{but} \mathrm{almost} \mathrm{no} \mathrm{staining} \mathrm{in} P t c h 1^{+/-}$and $\mathrm{Ptch}^{+/+}$animals, as shown in Figure 4D.

Expression of PCNA in UVB-irradiated skin in Ptch1 $1^{+/-} / A Z \operatorname{TgN}$ mice. Immunohistochemical staining for PCNA was used to detect proliferating cells in $\mathrm{Ptch}^{+/-} / \mathrm{AZ} \mathrm{TgN}$ and $P t c 1^{+/-}$mice. PCNA-positive nuclei were seen in the suprabasal epidermal layers of UVB-irradiated skin of $P t c h 1^{+/}$mice but were virtually absent in the skin of $P t c h 1^{+/-} / \mathrm{AZ} \operatorname{TgN}$ animals. Interestingly, microscopic BCCs in $P t c h 1^{+/-} / \mathrm{AZ}$ mice also showed diminished immunostaining for PCNA (Figure 4E).

\section{Studies in UVB-irradiated Ptch1 ${ }^{+/-}$heterozygous mice treated with DFMO}

To further document the effects of inhibition of ODC activity on the growth of UVB-induced BCCs, we employed DFMO, a suicidal ODC inhibitor. Oral administration of DFMO has been shown to suppress the development of a number of tumor types in various murine cancer models $(39,40)$, including UVB-induced SCCs in the skin of SKH-1 hairless mice $(35,41-44)$.
Effect of oral administration of DFMO on UVB-induced BCC development in Ptch $1^{+-}$mice. As shown in Figure 5, A and B, DFMO treatment reduced the total number of visible tumors by about 30\%. However, the number of BCCs and SCCs in the DFMO-treated animals was reduced to about $60 \%$ of that in the vehicle-treated control group, whereas there was no effect on the number of fibrosarcomas (Figure 5C). These tumor numbers represent the sum of both the already existing tumors at the beginning of the experiment and the new tumors that developed during the 20 weeks of DFMO treatment. It was not possible to ascertain whether DFMO treatment preferentially ablated existing tumors or whether it reduced new tumors, or both. As shown in Figure 5, D and E, DFMO treatment not only reduced visible tumors but also diminished BCC-like microscopic lesions by more than $80 \%$.

Effects of DFMO on the expression of SHH pathway genes. The effect of DFMO treatment on the expression of SHH pathway genes in non-tumor-bearing UVB-irradiated skin of $P t c h 1^{+/-}$mice was also determined. DFMO treatment reduced mRNA expression of the SHH pathway genes Ptch1, Ptch2, Gli1, Gli2, and Gli3 (data not shown). Effects of DFMO on the expression of cell cycle regulatory proteins. At week 5 of DFMO treatment, there was a substantial decrease in UVBinduced overexpression of cell cycle proteins that regulate proliferation, such as cyclins A2, B1, and D1 (Figure 6, A and B). However, in samples taken at the termination of the experiments (20 weeks of DFMO treatment), a similar reduction in cell cycle proteins was seen only in nontumor skin (Figure 6B). The expression of cyclin D1 mRNA was also decreased (data not shown). The diminished expression of cyclins in tumor tissue suggests the possibility that some tumors in these mice were resistant to DFMO.

Effects of DFMO on PCNA staining in UVB-induced BCCs. As shown in Figure $6 \mathrm{C}$, the strongly positive PCNA staining that characterizes BCCs and UVB-irradiated epidermis is largely abrogated in animals treated with DFMO. 
A

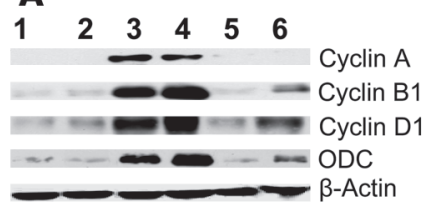

B

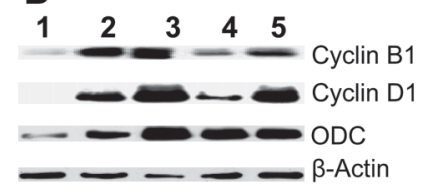

C

UVB
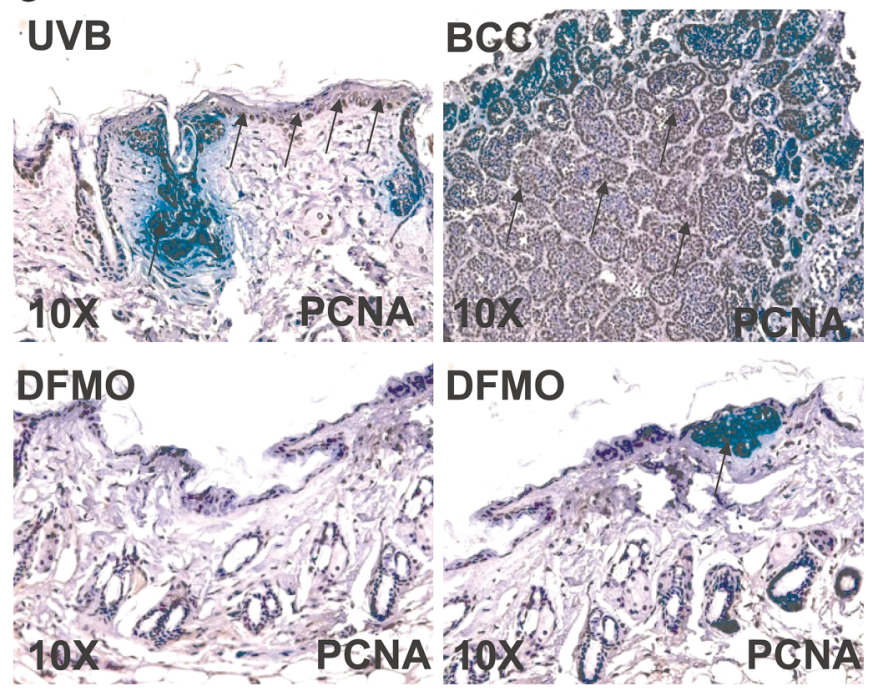

Figure 6

Effects of orally administered DFMO on UVB-induced expression of cell cycle regulatory proteins and proliferation markers in skin of Ptch $1^{+/-}$ mice. (A) Western blot showing the effects of orally administered DFMO on UVB-induced expression of cell cycle regulatory proteins in skin of Ptch1+/- mice. Samples were taken after 37 weeks, including 32 weeks of UVB irradiation followed by 5 weeks of DFMO treatment. Lanes 1 and 2 , age-matched nonirradiated normal skin (control); lanes 3 and 4, UVBirradiated nontumor skin; lanes 5 and 6, UVB-irradiated DFMO-treated nontumor skin. (B) Western blot showing the effects of orally administered DFMO on UVB-induced expression of cell cycle proteins in both skin and tumor of $P$ tch $1^{+/-}$mice. Samples were taken at the termination of the experiment at week 52. Lane 1, age-matched nonirradiated normal skin (control); lane 2, UVB-irradiated nontumor skin; lane 3, UVBinduced BCC; lane 4, UVB-irradiated DFMO-treated nontumor skin; lane 5, UVB-irradiated DFMO-treated BCC. (C) Immunohistochemical staining for PCNA in epidermis and BCCs in UVB-irradiated Ptch1+/- mice. The dark brown staining in the basal cell layer of epidermis and in tumor islands represents PCNA expression.

\section{Discussion}

Human cancers result largely from environmental exposures, and a major strategy for reducing cancer risk is to diminish the procarcinogenic effects of these exposures. This has led to a search for nontoxic chemical compounds capable of altering the activity of signaling pathways that drive the malignant phenotype $(45,46)$. ODC is an important enzyme in polyamine biosynthesis, which plays a crucial role in proliferative signaling and in carcinogenesis (18). In murine skin, ODC overexpression has been shown to promote the spontaneous development of SCCs in Hras transgenic mice (19). Administration of DFMO diminishes tumor development in this model and reduces UVB-induced SCCs in the skin of K5-ODC transgenic mice and in actinic keratoses in humans $(25,34,42)$. Increased ODC activity has been documented in sporadic human BCCs compared with adjacent nontumor skin, but little is known about ODC in the mul- tiple BCCs that develop in NBCCS patients. Our results indicate that overexpression of ODC enhances UVB-induced BCCs, whereas reduction of ODC expression, either by overexpression of $A Z$ or by administration of DFMO, substantially inhibits the development of these lesions in UVB-irradiated $P t c h 1^{+/-}$mice. This indicates the crucial importance of this enzyme in the pathogenesis of these tumors.

The K6 promoter in both $P t c h 1^{+/-} / \mathrm{ODC}$ TgN and $P t c h 1^{+/-} / \mathrm{AZ}$ TgN mice directs transgene overexpression to the ORS of hair follicles adjacent to the bulge region, where epidermal stem cells are believed to reside (47-49). It is likely that BCCs originate from stem cells in the ORS of hair follicles $(48,50)$. Thus, modulation of ODC expression in ORS keratinocytes could diminish their proliferative capacity directly, thereby reducing BCC tumorigenesis. It is also possible that inhibition of ODC in stromal cells could diminish the paracrine effects of polyamines in BCC tumorigenesis (12).

Our results confirm the importance of ODC as a proliferative signaling enzyme (51), since there was diminished PCNA staining in skin and tumors of $P t c h 1^{+/-} / \mathrm{AZ}$ TgN animals as well as $P t c h 1^{+/-}$ mice treated with DFMO. In contrast, PCNA and keratin 17 staining was enhanced in $P t c h 1^{+/} / \mathrm{ODC}$ TgN mice. It is of interest that ODC overexpression in these animals is associated with the apparent conversion of hair follicles into epidermal cysts, which show strongly positive immunostaining for ODC. This cystic transformation progresses with age and is followed by visible wrinkling and folding of the skin (Figure 1I). Enhanced epidermal cyst formation has also been observed in the skin of some NBCCS patients $(52,53)$. While ODC expression has not been assessed in the multiple BCCs in NBCCS patients (27), it seems highly likely that these lesions will manifest increased expression of this enzyme. A corollary to this hypothesis is that the epithelial lining of odontogenic keratinocysts in NBCCS patients has enhanced growth potential demonstrated by high expression of known proliferation markers such as PCNA and Ki67 (53-58).

It is conceivable that the use of genetic and pharmacologic approaches to the inhibition of ODC activity in tumor cells may also reduce enzyme activity in other tissues, leading to some adverse effects. Therefore, cancer-chemoprevention clinical trials that use abrogation of ODC activity should be planned carefully. However, it is encouraging that both murine and human consumption of DFMO in cancer-chemoprevention studies has revealed no toxic effects other than ototoxicity at high doses, which was reversible after discontinuation of DFMO treatment $(39,40)$. Furthermore, a topical formulation of DFMO (13.9\%) has been approved by the US Food and Drug Administration for human use for hair removal. Clinical studies have shown that this formulation has a favorable human dermal safety profile appropriate for a topical medication to be applied routinely (59). In addition, a phase II randomized trial of topical DFMO in human subjects reduced the number of precancerous actinic keratoses, suppressed polyamines, and reduced p53 protein. No serious adverse cutaneous effects were seen in these subjects (46).

The mechanism by which sustained activation of ODC drives the development of BCCs is unknown. However, Xie et al. showed that Gli1 can activate PDGFR $\alpha$ (60). Increased expression of PDGFR $\alpha$ was also observed in murine and human BCCs (60). PDGF-dependent signaling is known to activate various cell-proliferation pathways, including ODC activity (61). This is particularly important since it has recently been shown that BCCs lose PKC $\alpha$ expression and also, to some extent, PKC $\delta$ expression, which are essential for upregulation of Gli (62). These PKC isozymes are also known to reg- 
ulate the expression of ODC in response to tumor promoters (51). These data indicate that multiple mechanisms may be involved in regulating ODC levels in BCCs.

Altered regulation of cell cycle progression is associated with the development of epithelial cancers in many tissues, including skin. It is known that ODC activity is elevated in the late G1 phase and is associated with entry into the $S$ phase in various cell types (63). However, the impact of ODC overexpression on cell cycle progression is largely unknown. ODC overexpression in K6-ODC transgenic mice was shown to stimulate cyclin $\mathrm{E} / \mathrm{cdk} 2$ - and cyclin $\mathrm{A} / \mathrm{cdk} 2$-associated kinase activity, which in ODC/ras double-transgenic mice augments induction of cutaneous SCCs (64). Our results confirm that ODC overexpression is associated with elevated cyclin D1 and PCNA expression in BCCs induced by UVB in these animals. In Ptch1+/-/ODC TgN mice, we observed nuclear accumulation of cyclin D1 in BCC tumor cells, suggesting that cells transiting from the G0 phase remain in the G1 phase. The fact that DFMO treatment diminishes the expression of cyclins, including cyclin D1, suggests that ODC activity helps to drive rapid progression of the cell cycle in BCCs. Furthermore, the observed increase in PCNA staining in $P t c h 1^{+/-} / \mathrm{ODC}$ TgN mice and the decrease in both $P t c h 1^{+/-} / \mathrm{AZ} \mathrm{TgN}$ and DFMO-treated $P t c h 1^{+/-}$mice provide additional support for the concept that ODC and elevated polyamines augment cell proliferation in BCCs.

In summary, we have developed an innovative mouse model for the study of the pathogenesis of BCCs. We have established that our Ptch $1^{+/} /$ODC TgN mice manifest accelerated development of large numbers of BCCs, mimicking the phenotype of patients with NBCCS. These tumors closely resemble human BCCs, showing gross characteristic histologic features such as palisading basaloid cells in the periphery of tumor nests, the appearance of retraction spaces, the lack of keratinizing centers, and the characteristic expression pattern of marker biomolecules such as keratin 17, Gli1, Gli13, Ptch1, and Ptch2 $(65,66)$. In addition, these mice developed trichoblastomas, SCCs, and fibrosarcomas. Parental Ptch1 $1^{+/-}$mice spontaneously develop relatively few microscopic BCCs and trichoblastomas, and macroscopic tumor growth is minimal unless the animals are exposed to additional carcinogenic stimuli such as ionizing or UVB radiation. Using a standard UVB irradiation protocol, macroscopic BCCs are readily induced in $P t c h 1^{+/}$mice after 52 weeks of irradiation. However, our Ptch1 $1^{+/} /$ODC TgN mice showed accelerated spontaneous development of BCCs that was further augmented by UVB radiation, leading to a tumorigenic response that closely mimicked the phenotype of NBCCS patients. Our results indicate that inhibition of ODC activity either by genetic or by pharmacologic mechanisms substantially diminishes the development of BCCs. It is likely that chemopreventive agents capable of inhibiting ODC in our murine models could prove to be effective antitumor agents in human populations as well.

\section{Acknowledgments}

This work was supported by NIH grants N01 CN15109, R01 CA97249-01, NIH/NCI U19 CA81888, and NIAMS P30 AR44535 to D.R. Bickers and E.H. Epstein, Jr., and by CA-18138 to A.E. Pegg. The authors wish to thank Suzie Sass-Kuhn for the polyamine analysis.

Received for publication December 4, 2003, and accepted in revised form January 12, 2004.

Address correspondence to: Mohammad Athar, Department of Dermatology, College of Physicians and Surgeons, Columbia University, 630 West 168th Street, New York, New York 10032, USA. Phone: (212) 305-7618; Fax: (212) 305-7391; E-mail: ma493@columbia.edu.
1. Miller, D.L., and Weinstock, M.A. 1994. Nonmelanoma skin cancer in the United States: incidence. J. Am. Acad. Dermatol. 30:774-778.

2. Dlugosz, A., Merlino, G., and Yuspa, S.H. 2002. Progress in cutaneous cancer research. J. Investig. Dermatol. Symp. Proc. 7:17-26.

3. Giglia-Mari, G., and Sarasin, A. 2003. TP53 mutations in human skin cancers. Hum. Mutat. 21:217-228.

4. Gorlin, R.J. 1987. Nevoid basal-cell carcinoma syndrome. Medicine (Baltimore). 66:98-113.

5. Daya-Grosjean, L., and Sarasin, A. 2000. UV-specific mutations of the human patched gene in basal cell carcinomas from normal individuals and xeroderma pigmentosum patients. Mutat. Res. 450:193-199.

6. Bodak, N., et al. 1999. High levels of patched gene mutations in basal-cell carcinomas from patients with xeroderma pigmentosum. Proc. Natl. Acad. Sci. U. S. A. 96:5117-5122.

7. Bonifas, J.M., et al. 2001. Activation of expression of hedgehog target genes in basal cell carcinomas. J. Invest. Dermatol. 116:739-742.

8. Couve-Privat, S., Bouadjar, B., Avril, M.F., Sarasin, A., and Daya-Grosjean, L. 2002. Significantly high levels of ultraviolet-specific mutations in the smoothened gene in basal cell carcinomas from DNA repair-deficient xeroderma pigmentosum patients. Cancer Res. 62:7186-7189.

9. Gailani, M.R., and Bale, A.E. 1999. Acquired and inherited basal cell carcinomas and the patched gene. Adv. Dermatol. 14:261-283.

10. Xie, J., et al. 1998. Activating Smoothened mutations in sporadic basal-cell carcinoma. Nature. 391:90-92.

11. Saldanha, G. 2001. The Hedgehog signalling pathway and cancer. J. Pathol. 193:427-432.

12. Bale, A.E., and Yu, K.P. 2001. The hedgehog pathway and basal cell carcinomas. Hum. Mol. Genet. 10:757-762.
13. Sarasin, A. 1999. The molecular pathways of ultraviolet-induced carcinogenesis. Mutat. Res. 428:5-10.

14. Goodrich, L.V., Milenkovic, L., Higgins, K.M., and Scott, M.P. 1997. Altered neural cell fates and medulloblastoma in mouse patched mutants. Science. 277:1109-1113.

15. Aszterbaum, M., Beech, J., and Epstein, E.H., Jr. 1999. Ultraviolet radiation mutagenesis of hedgehog pathway genes in basal cell carcinomas. J. Investig. Dermatol. Symp. Proc. 4:41-45.

16. Aszterbaum, M., et al. 1999. Ultraviolet and ionizing radiation enhance the growth of BCCs and trichoblastomas in patched heterozygous knockout mice. Nat. Med. 5:1285-1291.

17. Pegg, A.E. 1988. Polyamine metabolism and its importance in neoplastic growth and a target for chemotherapy. Cancer Res. 48:759-774.

18. Pegg, A.E., et al. 2003. Transgenic mouse models for studies of the role of polyamines in normal, hypertrophic and neoplastic growth. Biochem. Soc. Trans. 31:356-360

19. Smith, M.K., Trempus, C.S., and Gilmour, S.K. 1998 Co-operation between follicular ornithine decarboxylase and v-Ha-ras induces spontaneous papillomas and malignant conversion in transgenic skin. Carcinogenesis. 19:1409-1415.

20. Scalabrino, G., et al. 1980. Levels of activity of the polyamine biosynthetic decarboxylases as indicators of degree of malignancy of human cutaneous epitheliomas. J. Invest. Dermatol. 74:122-124.

21. O'Brien, T.G., Simsiman, R.C., and Boutwell, R.K. 1975. Induction of the polyamine-biosynthetic enzymes in mouse epidermis by tumor-promoting agents. Cancer Res. 35:1662-1670.

22. Clifford, A., Morgan, D., Yuspa, S.H., Soler, A.P., and Gilmour, S. 1995. Role of ornithine decarboxylase in epidermal tumorigenesis. Cancer Res. 55:1680-1686.
23. O'Brien, T.G., Megosh, L.C., Gilliard, G., and Soler, A.P. 1997. Ornithine decarboxylase overexpression is a sufficient condition for tumor promotion in mouse skin. Cancer Res. 57:2630-2637.

24. Megosh, L.C., Hu, J., George, K., and O'Brien, T.G. 2002. Genetic control of polyamine-dependent susceptibility to skin tumorigenesis. Genomics. 79:505-512.

25. Ahmad, N., Gilliam, A.C., Katiyar, S.K., O’Brien, T.G., and Mukhtar, H. 2001. A definitive role of ornithine decarboxylase in photocarcinogenesis. Am. J. Pathol. 159:885-892.

26. Gokmen, S.S., Aygit, A.C., Ayhan, M.S., Yorulmaz, F., and Gulen, S. 2001. Significance of arginase and ornithine in malignant tumors of the human skin. J. Lab. Clin. Med. 137:340-344.

27. Kagoura, M., Toyoda, M., Matsui, C., and Morohashi, M. 2000. Immunohistochemical localization of ornithine decarboxylase in skin tumors. J. Cutan. Pathol. 27:338-343.

28. Hillary, R.A., and Pegg, A.E. 2003. Decarboxylases involved in polyamine biosynthesis and their inactivation by nitric oxide. Biochim. Biophys. Acta. 1647:161-166.

29. Coffino, P. 2001. Regulation of cellular polyamines by antizyme. Nat. Rev. Mol. Cell Biol. 2:188-194.

30. Hayashi, S., Murakami, Y., and Matsufuji, S. 1996. Ornithine decarboxylase antizyme: a novel type of regulatory protein. Trends Biochem. Sci. 21:27-30.

31. Feith, D.J., Shantz, L.M., and Pegg, A.E. 2001. Targeted antizyme expression in the skin of transgenic mice reduces tumor promoter induction of ornithine decarboxylase and decreases sensitivity to chemical carcinogenesis. Cancer Res. 61:6073-6081.

32. Kim, A.L., Athar, M., Bickers, D.R., and Gautier, J. 2002. Stage-specific alterations of cyclin expression during UVB-induced murine skin tumor development. Photochem. Photobiol. 75:58-67. 
33. Zhao, J., et al. 1999. Photoprotective effect of black tea extracts against UVB-induced phototoxicity in skin. Photochem. Photobiol. 70:637-644.

34. Fischer, S.M., Lee, M., and Lubet, R.A. 2001. Difluoromethylornithine is effective as both a preventive and therapeutic agent against the development of UV carcinogenesis in SKH hairless mice. Carcinogenesis. 22:83-88.

35. Smith, M.K., et al. 1997. Ornithine decarboxylase overexpression leads to increased epithelial tumor invasiveness. Cancer Res. 57:2104-2108.

36. Grachtchouk, M., et al. 2000. Basal cell carcinomas in mice overexpressing Gli2 in skin. Nat. Genet. 24:216-217.

37. Pegg, A.E., Shantz, L.M., and Coleman, C.S. 1995. Ornithine decarboxylase as a target for chemoprevention. J. Cell. Biochem. Suppl. 22:132-138.

38. Ramirez, A., Vidal, M., Bravo, A., and Jorcano, J.L. 1998. Analysis of sequences controlling tissue-specific and hyperproliferation-related keratin 6 gene expression in transgenic mice. DNA Cell Biol. 17:177-185.

39. Seiler, N. 2003. Thirty years of polyamine-related approaches to cancer therapy. Retrospect and prospect. Part 2. Structural analogues and derivatives. Curr. Drug Targets. 4:565-585.

40. Seiler, N. 2003. Thirty years of polyamine-related approaches to cancer therapy. Retrospect and prospect. Part 1. Selective enzyme inhibitors. Curr. Drug Targets. 4:537-564.

41. Arbeit, J.M., et al. 1999. Difluoromethylornithine chemoprevention of epidermal carcinogenesis in K14-HPV16 transgenic mice. Cancer Res. 59:3610-3620.

42. Carbone, P.P., et al. 1998. Phase I chemoprevention study of piroxicam and alpha-difluoromethylornithine. Cancer Epidemiol. Biomarkers Prev. 7:907-912.

43. Carbone, P.P., et al. 2001. Phase I chemoprevention study of difluoromethylornithine in subjects with organ transplants. Cancer Epidemiol. Biomarkers Prev. 10:657-661.

44. Shantz, L.M., Guo, Y., Sawicki, J.A., Pegg, A.E., and O'Brien, T.G. 2002. Overexpression of a dominantnegative ornithine decarboxylase in mouse skin: effect on enzyme activity and papilloma formation. Carcinogenesis. 23:657-664.

45. Bickers, D.R., and Athar, M. 2000. Novel approaches to chemoprevention of skin cancer. J. Dermatol. 27:691-695.

46. Einspahr, J.G., Bowden, G.T., and Alberts, D.S. 2003. Skin cancer chemoprevention: strategies to save our skin. Recent Results Cancer Res. 163:151-164.

47. Cotsarelis, G., Sun, T.T., and Lavker, R.M. 1990. Label-retaining cells reside in the bulge area of pilosebaceous unit: implications for follicular stem cells, hair cycle, and skin carcinogenesis. Cell. 61:1329-1337.

48. Owens, D.M., and Watt, F.M. 2003. Contribution of stem cells and differentiated cells to epidermal tumours. Nat. Rev. Cancer. 3:444-451.

49. Taylor, G., Lehrer, M.S., Jensen, P.J., Sun, T.T., and Lavker, R.M. 2000. Involvement of follicular stem cells in forming not only the follicle but also the epidermis. Cell. 102:451-461.

50. Alonso, L., and Fuchs, E. 2003. Stem cells of the skin epithelium. Proc. Natl. Acad. Sci. U. S. A. 100(Suppl. 1):11830-11835.

51. Wheeler, D.L., Ness, K.J., Oberley, T.D., and Verma, A.K. 2003. Inhibition of the development of metastatic squamous cell carcinoma in protein kinase $\mathrm{C}$ epsilon transgenic mice by alpha-difluoromethylornithine accompanied by marked hair follicle degeneration and hair loss. Cancer Res. 63:3037-3042.

52. Bale, S.J., Falk, R.T., and Rogers, G.R. 1998. Patching together the genetics of Gorlin syndrome. J. Cutan. Med. Surg. 3:31-34.

53. el Murtadi, A., Grehan, D., Toner, M., and McCartan, B.E. 1996. Proliferating cell nuclear antigen staining in syndrome and nonsyndrome odontogenic keratocysts. Oral Surg. Oral Med. Oral Pathol. Oral Radiol. Endod. 81:217-220.

54. Li, T.J., Browne, R.M., and Matthews, J.B. 1995. Epithelial cell proliferation in odontogenic keratocysts: a comparative immunocytochemical study of Ki67 in simple, recurrent and basal cell naevus syndrome (BCNS)-associated lesions. J. Oral Pathol. Med. 24:221-226.
55. Maiolino, P., Restucci, B., and De Vico, G. 1995 Expression of proliferating cell nuclear antigen in basal cell carcinomas and in squamous cell carcinomas of canine skin: correlation with mitotic index and histological features. Zentralbl. Veterinarmed. A. 42:339-343.

56. Barreto, D.C., Bale, A.E., De Marco, L., and Gomez, R.S. 2002. Immunolocalization of PTCH protein in odontogenic cysts and tumors. J. Dent. Res. 81:757-760.

57. Barreto, D.C., Gomez, R.S., Bale, A.E., Boson, W.L., and De Marco, L. 2000. PTCH gene mutations in odontogenic keratocysts. J. Dent. Res. 79:1418-1422.

58. Toth, D.P., Guenther, L.C., and Shum, D.T. 1996. Proliferating cell nuclear antigen (PCNA): prognostic value in the clinical recurrence of primary basal cell carcinoma. J. Dermatol. Sci. 11:36-40.

59. Hickman, J.G., Huber, F., and Palmisano, M. 2001. Human dermal safety studies with eflornithine $\mathrm{HCl}$ $13.9 \%$ cream (Vaniqa), a novel treatment for excessive facial hair. Curr. Med. Res. Opin. 16:235-244.

60. Xie, J., et al. 2001. A role of PDGFRalpha in basal cell carcinoma proliferation. Proc. Natl. Acad. Sci. U. S. A. 98:9255-9259.

61. Thyberg, J., and Fredholm, B.B. 1987. Induction of ornithine decarboxylase activity and putrescine synthesis in arterial smooth muscle cells stimulated with platelet-derived growth factor. Exp. Cell Res. 170:160-169.

62. Neill, G.W., et al. 2003. Loss of protein kinase Calpha expression may enhance the tumorigenic potential of Gli1 in basal cell carcinoma. Cancer Res. 63:4692-4697.

63. Kaczmarek, L., Calabretta, B., Ferrari, S., and de Riel, J.K. 1987. Cell-cycle-dependent expression of human ornithine decarboxylase. J. Cell. Physiol. 132:545-551.

64. Gilmour, S.K., Birchler, M., Smith, M.K., Rayca, K., and Mostochuk, J. 1999. Effect of elevated levels of ornithine decarboxylase on cell cycle progression in skin. Cell Growth Differ. 10:739-748.

65. Epstein, E., Jr. 2001. Genetic determinants of basal cell carcinoma risk. Med. Pediatr. Oncol. 36:555-558.

66. Wicking, C., Smyth, I., and Bale, A. 1999. The hedgehog signalling pathway in tumorigenesis and development. Oncogene. 18:7844-7851. 